\title{
A trans-dimensional approach to the behavioral aspects of depression
}

\section{João M. Bessa' , Ana R. Mesquita', Mário Oliveira', José Miguel Pêgo' ${ }^{1}$ João J. Cerqueira' ${ }^{1}$ Joana A. Palha', Osborne F. X. Almeida ${ }^{2}$ and Nuno Sousa ${ }^{1 *}$}

1 Life and Health Science Research Institute, School of Health Sciences, University of Minho, Braga, Portugal

2 Max Planck Institute of Psychiatry, Munich, Germany

Edited by:

David M. Diamond, University of South

Florida, USA

Reviewed by:

Therese Jay, INSERM U894, France

R.M. Tordera, Universidad de Navarra,

Spain

\section{*Correspondence:}

Nuno Sousa, Life and Health Science Research Institute, School of Health

Sciences, University of Minho,

Campus de Gualtar, 4710-057 Braga,

Portugal.

e-mail:njcsousa@ecsaude.uminho.pt
Depression, a complex mood disorder, displays high comorbidity with anxiety and cognitive disorders. To establish the extent of inter-dependence between these behavioral domains, we here undertook a systematic analysis to establish interactions between mood lassessed with the forced-swimming (FST) and sucrose consumption tests (SCT)], anxiety [elevatedplus maze (EPM) and novelty suppressed feeding (NSF) tests] and cognition (spatial memory and behavioral flexibility tests) in rats exposed to unpredictable chronic-mild-stress (uCMS). Expectedly, uCMS induced depressive-like behavior, a hyperanxious phenotype and cognitive impairment; with the exception of the measure of anxiety in the EPM, these effects were attenuated by antidepressants (imipramine, fluoxetine). Measures of mood by the FST and SCT were strongly correlated, whereas no significant correlations were found between the different measures of anxiety (EPM and NSF); likewise, measures of cognition by spatial memory and behavioral flexibility tests were poorly correlated. Inter-domain analysis revealed significant correlations between mood (FST and SCT) and anxiety-like behavior (NSF, but not EPM). Furthermore, significant correlations were found between cognitive performance (reverse learning task) and mood (FST and SCT) and anxiety-like behavior (NSF). These results demonstrate interactions between different behavioral domains that crosscut the disciplines of psychiatry and neurology.

Keywords: depression, anxiety, cognition, antidepressants, stress, modelling

\section{INTRODUCTION}

Psychiatric disorders, including depression, are complex and heterogeneous clinical entities. In the absence of well defined pathophysiological factors at the cellular and molecular level, the clinical definition and classification of depression has been structured in diagnostic tools such as the Diagnostic and Statistical Manual of Mental Disorders (DSM-IV) and the International Classification of Diseases and Related Health Problems (ICD-10). This syndromatic approach relies in the clustering of core psychopathological phenomena that characterize a depressive state. However, the assumed core symptoms, such as anhedonia and learned helplessness, are not specific, being often present in distinct psychiatric disorders such as bipolar disorder, obsessive compulsive disorder or even schizophrenia (Mathews et al., 2005).

The interesting overlap between the emotional changes observed in depression and anxiety disorders suggest a common psychopathological pathway that may account for the high co-morbidity between mood and anxiety disorders (Hettema, 2008; Mergl et al., 2007). Less marked, but equally relevant, are the cognitive changes observed in depression and anxiety disorders (Castaneda et al., 2008), and the idea of a continuum between emotional changes and cognitive impairments has recently gained currency (Sotiropoulos et al., 2008; Swaab et al., 2005). Thus, the subjective separation of these three behavioral dimensions - depressive mood, anxiety and impaired cognition - while practical for diagnostic classification purposes and for their modelling, tends to hinder understanding of the individual and synergistic contributions of these dimensions to the overall features of the clinical disorder.

Animal models to study the etiopathogenic factors involved in depression and which are aimed at facilitating novel therapeutic strategies usually consider the disease from a monosymptomatic perspective. Each of the available models usually relies on a single behavioral phenotype that is theoretically related to a core depressive symptom (face validity) and which can be modulated by psychotropic drugs with antidepressive properties (predictive validity) (Fuchs and Flïugge, 2006; Kalueff et al., 2007). Despair paradigms such as the forced swimming (Cryan et al., 2005b) and tail suspension (Cryan et al., 2005a) tests are commonly used for verifying the potential efficacy of acutely and chronically administered antidepressants. These tests, which examine reversibility of the learned helplessness response that develops upon exposure to an inescapable stress, have, however, a serious flaw: drug efficacy is measured under basal conditions, i.e. in animals that do not necessarily display signs of depressive-like behavior, which seriously compromises their clinical therapeutic relevance (Cryan and Slattery, 2007). Models that somewhat overcome the problem of aetiological validity, include exposure to a paradigm in which the animal repeatedly fails to escape from an inescapable shock (learned helplessness) (Henn and Vollmayr, 2005), olfactory bulbectomy (Song and Leonard, 2005), and chronic exposure to stressors of variable 
quality, intensity and duration. Paradigms involving chronic stress, such as the sustained social stress (Fuchs, 2005) or unpredictable chronic mild stress (uCMS) (Porsolt, 2000; Willner and Mitchell, 2002), have produced the most consistent results in terms of anhedonia and learned helplessness, particularly in rats (D'Aquila et al., 1994; Willner, 2005). Importantly, the stress-induced depressivelike behavior that results from such treatments can be attenuated by various antidepressant drugs. Interestingly, independent studies have shown that chronic stress also triggers anxiety (Griebel et al., 2002a,b) and cognitive impairments in spatial memory tasks (Song et al., 2006) and object recognition tasks (Orsetti et al., 2007), which in the case of the latter can be ameliorated by antidepressant treatment (Elizalde et al., 2008). Despite the fact that anhedonic and learned helplessness responses to uCMS have been correlated in previous studies (Strekalova et al., 2004), we are unaware of any study designed to assess the interplay between mood, anxiety and cognition in the uCMS animal model of depression.

In this study, a depressive-like phenotype was induced in rats using an uCMS paradigm. Depressive-like behavior was confirmed with the sucrose consumption test (SCT) and forced swimming test (FST), anxiety-related behavior was evaluated in the elevated-plus maze (EPM) and novelty suppressed feeding (NSF) paradigm, and learning and memory acquisition were tested using two different tasks in the Morris water maze (MWM). In addition, the therapeutic effects of two different antidepressant drugs (fluoxetine and imipramine) on these three behavioral dimensions were evaluated.

\section{MATERIALS AND METHODS ANIMALS}

Male Wistar rats (Charles-River Laboratories), weighing 300-400 g and aged 3 months were housed (three per cage) under standard laboratory conditions ( $12 \mathrm{~h}$ light: $12 \mathrm{~h}$ dark cycle, at $22^{\circ} \mathrm{C}$, relative humidity of $55 \%$; free access to food and water). Fifty-six animals were randomly assigned to four main experimental groups - a control group without stress exposure, treated with saline and three groups exposed to CMS and treated with either saline, fluoxetine or imipramine ( $n=14$ per group). Animals were brought to the behavioral laboratory at least $1 \mathrm{~h}$ prior to testing and all experimental sessions were conducted during the diurnal phase, between 10:00 and 18:00 h. All procedures were carried out in accordance with European Union Directive 86/609/EEC and NIH guidelines on animal care and experimentation.

\section{UNPREDICTABLE CHRONIC MILD STRESS}

A slightly modified version of an uCMS protocol (Willner, 2005) was used. It consisted of chronic exposure to unpredictable mild stressors (confinement to a restricted space for $1 \mathrm{~h}$, placement in a tilted cage $\left(30^{\circ}\right)$ for $3 \mathrm{~h}$, housing on damp bedding for $8 \mathrm{~h}$, overnight illumination, $18 \mathrm{~h}$ food deprivation followed by exposure to inaccessible food for $1 \mathrm{~h}$, water deprivation for $18 \mathrm{~h}$ followed by exposure to an empty bottle for $1 \mathrm{~h}$, and reversed light/dark cycle for $48 \mathrm{~h}$ every 7 days) over a period of 6 weeks. During the last 2 weeks of uCMS, animals were given daily injections of saline, fluoxetine or imipramine.

All animals (controls and uCMS-exposed) were given a behavioral test battery, as described below. Sucrose preference tests were performed weekly over the 6 weeks of exposure to uCMS. The rest of the tests (EPM, NSF, FST and two learning tasks in the MWM) were administered sequentially according to a design to avoid between-test interference effects (Elizalde et al., 2008; Võikar et al., 2001).

\section{SUCROSE CONSUMPTION TEST}

Anhedonia was assessed weekly during exposure to uCMS using the SCT. Briefly, animals were allowed to habituate to the sucrose solution for 1 week before the uCMS protocol to establish baseline preference levels. To test sucrose preference, animals that were food- and water-deprived for $18 \mathrm{~h}$ were presented with two pre-weighed bottles containing 1\% sucrose solution or tap water for a period of $1 \mathrm{~h}$. Sucrose preference was calculated according to the formula: sucrose preference $=[$ sucrose intake $/$ (sucrose intake + water intake) $] \times 100$, as previously described (Bekris et al., 2005). Anhedonia was defined as a reduction in sucrose preference relative to baseline levels.

\section{ELEVATED-PLUS MAZE}

The EPM was used to test anxiety-like behavior. The EPM apparatus was made of black polypropylene (ENV-560; MedAssociates), consisted of two opposite open arms $(50.8 \times 10.2 \mathrm{~cm})$ and two enclosed arms $(50.8 \times 10.2 \times 40.6 \mathrm{~cm})$ elevated $72.4 \mathrm{~cm}$ above the floor. The junction area between the four arms measured $10 \times 10 \mathrm{~cm}$. A raised edge $(0.5 \mathrm{~cm})$ on the open arms provided additional grip for the rats. Rats were placed individually in the center of the maze facing a closed arm and were allowed 5 min of free exploration. Behavioral parameters were recorded with the use of an infra-red photobeam system connected to a computer with specific software (MedPCIV, MedAssociates). After each trial, the maze was cleaned with $10 \%$ ethanol. The time spent in the open arms was used to calculate the percentage of time in the open arms which was taken as an index of anxiety-like behavior. The total number of entries in the closed arms was used as a measure of general locomotor activity.

\section{NOVELTY SUPPRESSED FEEDING}

Anxiety-like behavior was also assessed using the NSF paradigm. Following published protocols (Bodnoff et al., 1998), animals were food-deprived for $23 \mathrm{~h}$ before being placed in a novel environment for $10 \mathrm{~min}$ (an open-field arena; MedAssociates Inc) where a single food pellet was centrally placed. Upon reaching the pellet, animals were returned to their home cages where they were presented with pre-weighed food over a period of $5 \mathrm{~min}$. Latency to feeding in the open field was used as an index of anxiety-like behavior; the amount of food consumed in the home cage provided a measure of appetitive drive.

\section{FORCED SWIMMING TEST}

Learned helplessness was evaluated in the FST on the last day of exposure to uCMS. Twenty-four hours after a pre-test session $(10 \mathrm{~min})$, rats were placed in cylinders filled with water $\left(25^{\circ} \mathrm{C}\right.$; depth $30 \mathrm{~cm}$ ) for a period of $5 \mathrm{~min}$. Test sessions were assessed using a camera connected to a video tracking system (Viewpoint); the system automatically calculated immobility time and latency to immobility. Learned helplessness behavior was defined as an increase in time of immobility and a decrease in latency to immobility. 


\section{MORRIS WATER MAZE}

An evaluation of cognitive function was performed in a spatial learning task (hippocampal-dependent) and a reverse learning task (prefrontal cortex-dependent) in the MWM (Cerqueira et al., 2007a). The MWM apparatus consisted of a black tank (diameter: $170 \mathrm{~cm}$; depth: $50 \mathrm{~cm}$ ), divided in quadrants by imaginary lines, and filled with water $\left(22^{\circ} \mathrm{C}\right)$ to a depth of $31 \mathrm{~cm}$. During testing, a black platform $(12 \times 12 \mathrm{~cm}$; invisible to the rats $)$ was placed at a height of $30 \mathrm{~cm}$. The room was dimly lit and extrinsic visual clues were glued to the walls. Data were collected using a videotracking system (Viewpoint). In the spatial learning task, animals were tested for three consecutive days (four trials per day, with a maximum of 2 min per trial). The escape platform was placed in the centre of an arbitrarily-defined quadrant, assigned to a specific test subject. To avoid possible bias effects due to geographic preference, "animal-platform position" was paired between the different treatment groups. Test sessions begun with rats being placed, facing the wall of the maze, in a defined start position and finished once the escape platform had been reached. This procedure was continued in a clock-wise fashion over the subsequent trials. The distance travelled and the time spent to reach the platform was recorded. When the escape platform was not reached within $2 \mathrm{~min}$, the experimenter guided the animal to the platform. At the end of each test session, animals were dried and allowed to rest for $30 \mathrm{~s}$ before being returned to the maze for the remaining test sessions of that day. On the fourth day, animals were tested in a reverse learning task in which the escape platform was positioned in a new (opposite) quadrant and rats were tested in a four-trial paradigm, as described above. For this task, distance and time spent swimming in each quadrant were recorded. The difference between distances travelled in the quadrant containing the newly-positioned platform ("new") and the quadrant that previously contained the platform ("old") was calculated as a measure of reversal performance. The total distance swum was evaluated as a measure of locomotor activity.

\section{DRUGS}

The antidepressant drugs used were fluoxetine $(10 \mathrm{mg} / \mathrm{kg}$, Kemprotec) and imipramine (10 mg/kg, Sigma-Aldrich). Compounds were dissolved in 5\% DMSO in $0.9 \%$ saline. Fluoxetine, imipramine and vehicle ( $0.9 \%$ saline) were administered intraperitoneally $(1 \mathrm{ml} / \mathrm{kg})$ during the last 2 weeks of the uCMS protocol. All injections were performed at 20:00. The doses of antidepressant drugs administered daily were chosen based in their therapeutic effects in previous studies (Song et al., 2006).

\section{STATISTICAL ANALYSIS}

After confirmation of homogeneity, appropriate statistical tests were applied to the data. Repeated measures ANOVA was used to analyze the results of the sucrose preference test and of the spatial learning task in the MWM. One-way ANOVA was used to evaluate the impact of $\mathrm{uCMS}$ and antidepressant treatment in the EPM, NSF, FST and reverse learning task in the MWM. Differences between groups were determined by post-hoc analysis, using Tukey's honestly significant difference test (Tukey HSD). Correlations between the different behavioral parameters were evaluated by the Pearson coefficient $(r)$ test in which data from control and uCMS groups was included. Statistical significance was accepted for $P<0.05$. Results are expressed as mean \pm s.e.m.

\section{RESULTS \\ PARALLEL CHANGES IN MOOD, ANXIETY AND COGNITION AFTER UCMS AND ANTIDEPRESSANTS}

Exposure to uCMS induced relevant changes in all three behavioral domains studied. In the SCT, performed during the 6 weeks of the protocol, animals exposed to uCMS showed a significantly decreased preference for sucrose when compared to control animals $\left(F_{1,26}=89.513, P<0.001\right)$ (Figure 1A). This effect was significantly reversed by antidepressant treatment $\left(F_{2,52}=5.559\right.$, $P=0.006)$. Curiously, the tricyclic agent imipramine exhibited an earlier onset of antidepressant action; this drug reversed anhedonic behavior within the first week of treatment $(P<0.001)$, whereas the responses to the SSRI fluoxetine only appeared after 2 weeks $(P<0.001)$. Consistent with these data suggestive of a depressed phenotype, animals exposed to uCMS showed increased immobility time $\left(F_{1,26}=37.568, P<0.001\right)$ (Figure 1B) and decreased latency to immobility $\left(F_{1,26}=32.894, P<0.001\right)$ in the FST (data not shown). Antidepressant treatment proved to be a significant factor in recovery from learned helplessness as evaluated in the FST $\left(F_{2,52}=27.962, P<0.001\right)$; when compared to stressed rats that did not receive drug treatments, immobility time was significantly reduced by fluoxetine $(P<0.001)$ and imipramine $(P<0.001)$ and the latency time to immobility was significantly increased by both drugs $(P<0.001)$ (data not shown).

As compared to controls, animals exposed to uCMS spent significantly less time in the open arms of the EPM $\left(F_{1,26}=4.405\right.$, $P=0.046$ ) (Figure 1C), but displayed no changes in locomotor activity (measured by the total number of closed arms entries) (data not shown). The performance in the EPM was not significantly affected by antidepressant treatment $\left(F_{2,52}=0.630, P=0.534\right)$. In the NSF test, stressed animals exhibited an increase in the latency time to feed $\left(F_{1,26}=6.177, P=0.02\right)$ (Figure 1D), while food intake did not differ between control and uCMS-exposed rats (data not shown). A significant effect of treatment was observed in the $\operatorname{NSF}\left(F_{2,52}=2.517\right.$, $P<0.001$ ), with $P<0.001$ in animals treated with either fluoxetine or imipramine vs. stressed animals receiving no drug treatment. Interestingly, imipramine in stressed animals proved to be anxiolytic in the NSF, when compared to control animals $(P=0.02)$. Neither antidepressant altered food intake in the NSF (data not shown).

Evaluation of spatial learning in the MWM failed to reveal any significant differences between controls and uCMS animals (Figure 2A). Accordingly, neither imipramine nor fluoxetine induced changes in performance in the spatial learning task in the MWM. On the other hand, performance in the reverse learning task was significantly impaired in animals exposed to uCMS (Figure 2B), indicated by the lower percentage of distance swum in the "new" quadrant to which the platform had been re-positioned $\left(F_{1,26}=13.427, P=0.001\right)$, as opposed to an higher percentage spent in the "old" quadrant containing the platform during training sessions $\left(F_{1,26}=12.716, P=0.001\right)$. This impairment in reverse learning was reversed by antidepressant treatment $\left(F_{2,52}=4.476, P=0.016\right.$, for the percentage of distance swum in quadrant containing the re-positioned platform and $F_{2,52}=9.021, P<0.001$ in the quadrant containing the platform during training sessions). Animals 

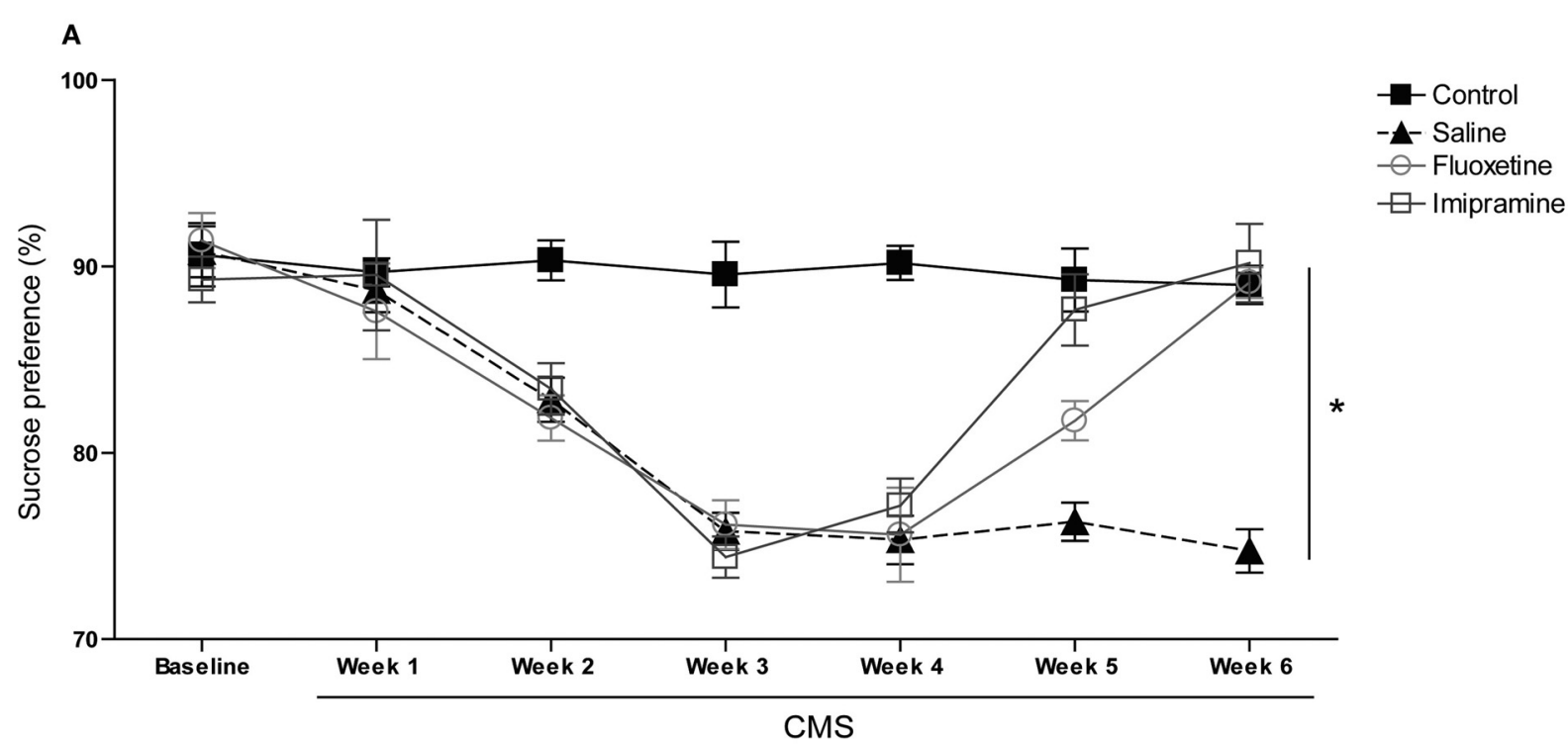

モImipramine

B

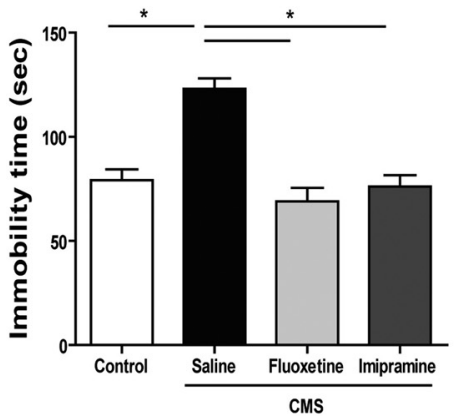

C

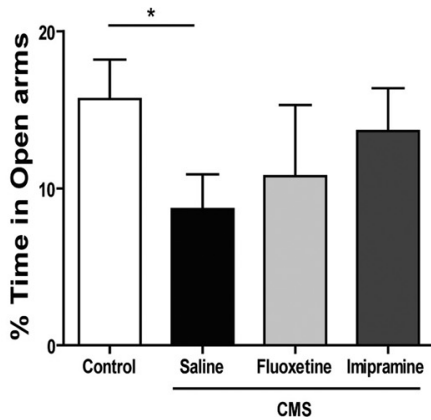

D

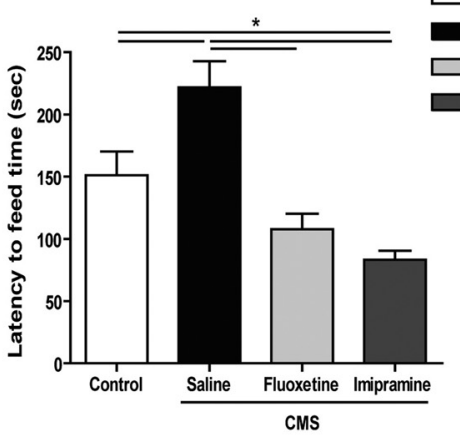

FIGURE 1 | Behavioral effects of uCMS and antidepressant treatment on mood and anxiety domains. (A) Sucrose preference in the SCTs performed during uCMS. (B) Immobility time in the FST, (C) percentage of time spent in the open arms of the EPM and (D) latency time to feed in the NSF paradigm. ${ }^{*} P<0.005$. Data presented as mean \pm s.e.m.

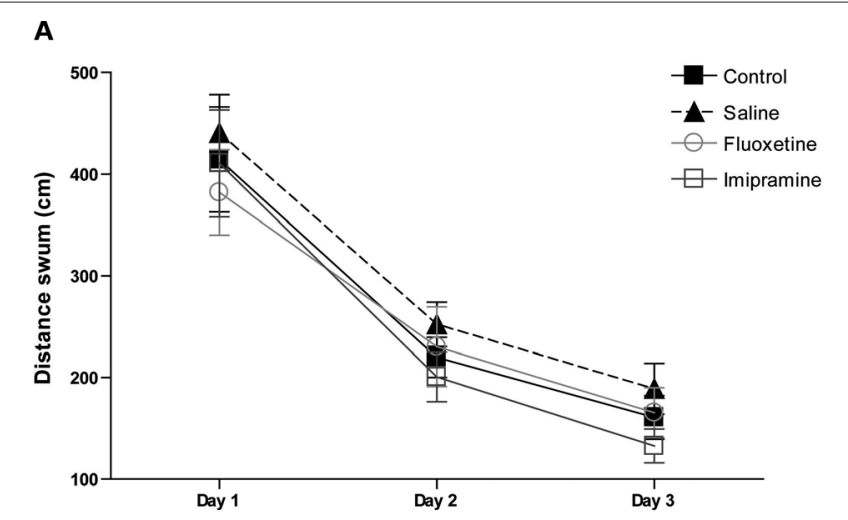

B

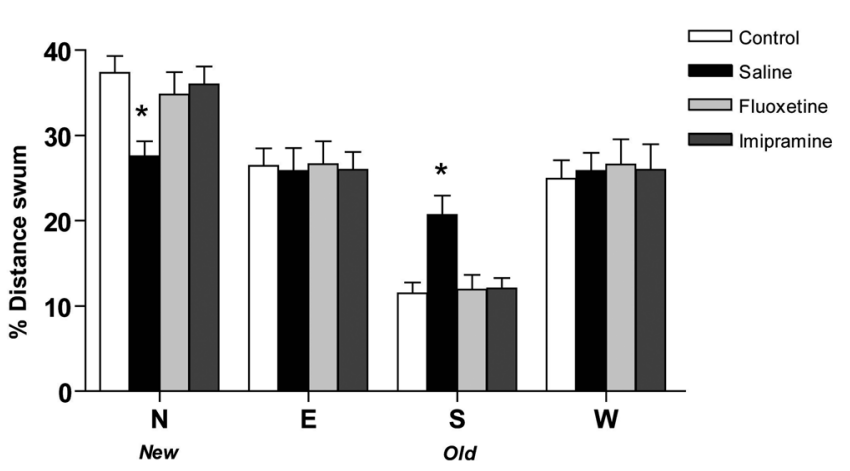

FIGURE 2 | Behavioral effects of antidepressants in the cognitive performance in the Morris watermaze. (A) Average distance swum in the spatial learning task and (B) the percentage of distance swum in each quadrant in the reverse learning task in the Morris watermaze. ${ }^{*} P<0.005$. Data presented as mean \pm s.em. 
treated with fluoxetine and imipramine travelled significantly lower distances in the "old" quadrant $(P=0.003$ for both $)$ and longer distances in the "new" quadrant $(P=0.046$ and $P=0.038$, respectively). Locomotor activity measured by the total distance swum was not altered by exposure to stress or treatment with antidepressants, confirming the results observed in the EPM.

\section{INTERPLAY BETWEEN STRESS-INDUCED CHANGES IN MOOD, ANXIETY AND COGNITION}

Intra-domain correlation analysis revealed a negative correlation between sucrose preference and immobility time in the FST ( $r=-0.751$ (26), $P<0.001)$, confirming that animals with lower preference for sucrose were immobile for longer in the FST. In contrast, no significant correlation was found between the measures of anxiety in the EPM and NSF, or the measures of cognition in the two versions of the MWM (spatial learning and reversal learning).

Correlation analysis was also performed to inspect for synergistic inter-domain effects as represented in Figure 3. Analyzing the interactions between mood and anxiety revealed a negative correlation between sucrose preference and latency of time to feed in the NSF $[r=-0.397$ (26), $P=0.036]$; in addition, a positive correlation between immobility time in the FST and latency of time to feed in the NSF $[r=0.458$ (26), $P=0.014]$ was found. On the other hand, we failed to find significant correlations between parameters of mood and percentage of time in the open arms of the EPM. With respect to interactions between mood and cognition, we observed a positive correlation between sucrose preference and performance in the reverse learning task $[r=0.695$ (26), $P<0.001]$; moreover, a negative correlation was found between immobility time in the FST and performance (in the reverse learning task $[r=-0.561$ (26), $P=0.002]$. Finally, an interplay between anxiety and cognition was demonstrated by the negative correlation between the latency of time to feed in the NSF and the performance in the reverse learning task $[r=-0.404(26), P=0.033]$.

\section{DISCUSSION}

This study used a multidimensional analysis of the behavioral responses to uCMS to examine the relevance of commonly used measures in this animal model of human depression. Etiological validity of the model is provided by the fact that stress is an important precipitating event both in the animal model and in clinical depression (Willner and Mitchell, 2002). Thus, the use of uCMS to model depressive-like behavior is justified. In addition, construct validity is conferred to the model by the fact that the neurobiological processes triggered by stress are similar in animals and humans (Anisman and Matheson, 2005; Steckler, 2001). Nevertheless, the uCMS model of depression has remained poorly characterized in terms of the similarities with the symptom profile of clinical depression, namely in its anxiety and cognitive dimensions (Willner, 2005). By demonstrating significant inter-domain behavioral interactions, the present work adds significantly to the face validity of the uCMS model of depression.

Our results confirm previous observations (Bekris et al., 2005; D’Aquila et al., 1994; Silva et al., 2008; Willner et al., 1996) that exposure to uCMS induces significant changes in mood-related behavior, characterized by an anhedonic state with decreased sucrose preference and increased immobility in the FST. Furthermore, the significant negative correlation observed between these two measures implies that the most anhedonic animals are the most helpless in the FST. This demonstration further strengthens the view that anhedonia and learned helplessness are core symptoms of depressive behavior.

The effect of uCMS in anxiety-related behavior has been a matter of recent debate (Kompagne et al., 2008), with reports of decreased (D’Aquila et al., 1994; Kopp et al., 1999) and increased (Griebel et al., 2002a,b; Maslova et al., 2002) anxiety-like behavior in the EPM test after uCMS. In the present study, animals exposed to uCMS displayed marginally significant decreases in the percentage of time spent in the open arms of the EPM, suggesting the induction of a mildly anxious phenotype. To overcome this lack of reproducibility in the EPM, we evaluated the impact of uCMS in another paradigm of anxiety-related behavior - the NSF paradigm (Bodnoff et al., 1998). Stress-induced anxiogenesis was confirmed in the NSF by the observation of a significant increase in the latency time to feeding. Since the results of the two tests of anxiety behavior (EPM and NSF) were not correlated, it would appear that each of these tests measures a distinct aspect of anxiety. In this respect, it should be noted that although both the EPM and

\section{A}

Correlations between behavioral measures

\begin{tabular}{|c|c|c|}
\hline Mood / Anxiety & $\%$ Open arm time & Latency to feed \\
\hline Immobility time & $r=-0.336, N S$ & $r=0.458, p=0.014$ \\
\hline Sucrose preference & $r=-0.314, N S$ & $r=-0.397, p=0.036$ \\
\hline Mood / Cognition & Distance Swam & New-Old quadrant \\
\hline Immobility time & $r=0.254, N S$ & $r=-0.561, p=0.002$ \\
\hline Sucrose preference & $r=-0.171, N S$ & $r=0.695, p<0.001$ \\
\hline Anxiety / Cognition & Distance Swam & New-Old quadrant \\
\hline$\%$ Open arm time & $r=0.007, N S$ & $r=0.331, N S$ \\
\hline Latency to feed & $r=0.294$, NS & $r=-0.404, p=0.033$ \\
\hline
\end{tabular}

B

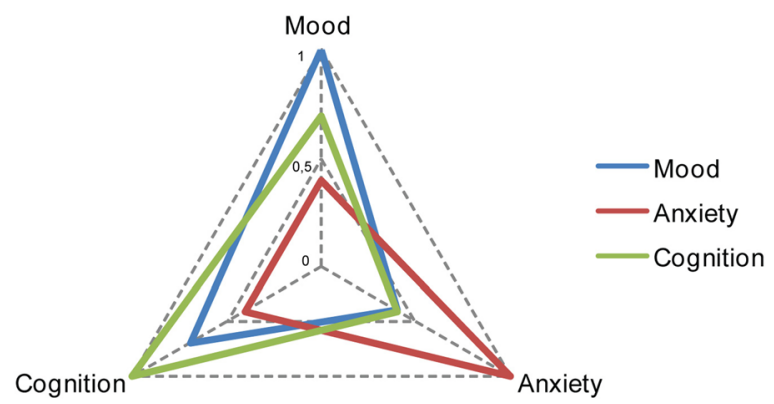

FIGURE 3 | Inter-domain correlations between mood, anxiety and cognition. (A) Table shows Pearson correlation coefficients between different behavioral parameters and (B) graphical representation of the correlations between mood (sucrose preference), anxiety (latency to feed) and cognition (new-old quadrant distances). 
NSF are conflict-based tests (Sousa et al., 2006), performance in the EPM depends strongly on exploratory behavior, contrasting with behavior in the NSF which depends on appetitive drive because of the necessity of food deprivation. Accordingly, behavior in the EPM may be more sensitive to minor differences in environmental stimuli, possibly accounting for the disparate reports on the effects of uCMS.

Several studies have described uCMS-induced changes in cognitive function. For example, mice subjected to the uCMS paradigm show impairments in recognition memory (Elizalde et al., 2008; Li et al., 2008) as well as in spatial learning and memory (Song et al., 2006). Our study failed to observe performance differences between stressed and control rats in a spatial learning task in the MWM. However, those animals exposed to uCMS displayed marked cognitive impairments when tested in a reverse learning task. Dissociation in cognitive performance in the different tasks may be suggestive of the greater vulnerability of the prefrontal cortex (a brain region implicated in reverse learning; Cerqueira et al., 2007a,b) to the deleterious effects of uCMS, as compared to the hippocampus (which is primarily involved in spatial learning tasks). Supporting this interpretation, the analysis revealed poor correlation between performances on the two tasks.

A primary aim of this study was to assess the complex interactions between mood, anxiety and cognition. Previous studies reported correlations between immobility time in the FST and performance in an object recognition task (Elizalde et al., 2008) and between sucrose preference and performance in a social avoidance test (Kompagne et al., 2008). Analysis of data collected in the present study revealed a strong correlation between the two core symptoms of depressive behavior - anhedonia and learned helplessness - and both, the anxious phenotype (measured in the NSF) and cognitive impairments (reverse learning task). Furthermore, a significant correlation emerged between anxiety-related behavior in the NSF and cognitive performance in the reverse learning task. These results therefore show that the three different behavioral dimensions analyzed are likely to be interdependent, with each contributing to the dynamic processes that underlie depressive behavior.

The issue of whether the uCMS paradigm represents a valid model of depression was addressed by analyzing the ability of antidepressant drugs to ameliorate the various uCMS-induced changes in behavior (predictive validity). Consistent with previous work (Griebel et al., 2002a; Song et al., 2006), the present experiments

\section{REFERENCES}

Anisman, H., and Matheson, K. (2005). Stress, depression, and anhedonia; Caveats concerning animal models. Neurosci. Biobehav. Rev. 29, 525-546.

Bekris, S., Antoniou, K., Daskas, S., and Papadopoulou-Daifoti, Z. (2005). Behavioral and neurochemical effects induced by chronic mild stress applied to two different rat strains. Behav. Brain. Res. 161, 45-59.

Bodnoff, S. R., Suranyi-Cadotte, B., Aitken, D. H., Quirion, R., and Meaney, M. J. (1998). The effects of chronic antidepressant treat-

demonstrated the efficacy of tricyclic agents (e.g. imipramine) and selective serotonin reuptake inhibitors (SSRI; e.g. fluoxetine) in reversing the anhedonic and helpless behaviors that develop after exposure to uCMS. Interestingly, imipramine showed a faster onset of action (with 1 week) as compared to fluoxetine whose first effects were observed only after 2 weeks; these different temporal profiles most likely reflect the dosages used, different mechanisms of drug action and/or neurochemical substrates.

The anxiolytic actions of antidepressant drugs have been extensively demonstrated in the NSF paradigm in stress-naive animals (Bodnoff et al., 1989) as well as in transgenic models of depressive behavior, e.g. the serotonin transporter (SERT) knockout rat (Olivier et al., 2008). In the present study, we found that both fluoxetine and imipramine displayed anxiolytic properties when uCMS-exposed rats were tested in the NSF, but not in the EPM. These findings reinforce the view that the EPM, although considered to be the gold standard in the development of novel anxiolytic agents (Dawson and Tricklebank, 1995), may lack the desired sensitivity and reproducibility in stress exposure models and chronic antidepressant treatment.

Finally, our results clearly revealed that imipramine and fluoxetine attenuate uCMS-induced impairments in cognitive performance, as measured by the reverse learning task in the MWM. These findings concur with those of a previous study that reported partial reversal by the SSRI paroxetine of stress-induced impairment in an object recognition task (Elizalde et al., 2008), as well as those of a recent clinical study that suggested the use of antidepressants for improving prefrontal cortex-dependent cognitive functions in patients with recurrent depression (Borkowska et al., 2007).

In summary, the results of this study show that depression-like behavior, anxiety and impaired cognition as three highly interdependent variables that act in overlapping and synergistic modes; from a broader viewpoint, our results also show that there is a continuum between emotional changes and cognitive impairments. Further, these results demonstrate interactions between different behavioral domains that crosscut the disciplines of psychiatry and neurology.

\section{ACKNOWLEDGMENTS}

This project was supported by the Portuguese Foundation for Science and Technology (FCT) (PTDC/SAU-NEU/72699/2006) and the European Commission (FP 7 - CRESCENDO - Contract LSHM-CT-2005-018652).

patients with recurrent depression. Neuropsychopharmacol. Hung. 9, 131-136.

Castaneda, A. E., Tuulio-Henriksson, A. Marttunen, M., Suvisaari, J., and Lönnqvist, J. (2008). A review on cognitive impairments in depressive and anxiety disorders with a focus on young adults. J. Affect Disord. 106, $1-27$.

Cerqueira, J. J., Mailliet, F., Almeida, O. F., Jay, T. M., and Sousa, N. (2007a). The prefrontal cortex as a key target of the maladaptive response to stress. J. Neurosci. 27, 2781-2787.
Cerqueira, J. J., Taipa, R., Uylings, H. B., Almeida, O. F., and Sousa, N. (2007b). Specific configuration of dendritic degeneration in pyramidal neurons of the medial prefrontal cortex induced by differing corticosteroid regimens. Cereb. Cortex 17, 1998-2006.

Cryan,J.F.,Mombereau,C., andVassout, A. (2005a). The tail suspension test as a model for assessing antidepressant activity: review of pharmacological and genetic studies in mice. Neurosci. Biobehav. Rev. 29, 571-625.

(2007). Enhancing effect of mirtazapine on cognitive functions associated with prefrontal cortex in
Cryan, J. F., and Slattery, D. A. (2007). Animal models of mood disorders: 
recent developments. Curr. Opin. Psychiatry 20, 1-7.

Cryan, J. F., Valentino, R. J., and Lucki, I. (2005b). Assessing substrates underlying the behavioral effects of antidepressants using the modified rat forced swimming test. Neurosci. Biobehav. Rev. 29, 547-569.

D’Aquila, P., Brain, P. F., and Willner, P. (1994). Effects of chronic mild stress on performance in behavioral tests relevant to anxiety and depression. Physiol. Behav. 56, 861-867.

Dawson, G. R., and Tricklebank, M. D. (1995). Use of elevated-plus-maze in the search for novel anxiolytic agents. Trends Pharmacol. Sci. 16, 33-36.

Elizalde, N., Gil-Bea, F. J., Ramírez, M. J., Aisa, B., Lasheras, B., et al. (2008). Longlasting behavioral effects and recognition memory deficit induced by chronic mild stress in mice: effect of antidepressant treatment. Psychopharmacology (Berl). 199, 1-14.

Fuchs, E. (2005). Social stress in tree shrews as an animal model of depression: an example of a behavioral model of a CNS disorder. CNS Spectr. $10,182-190$

Fuchs, E., and Flïugge, G. (2006). Experimental animal models for the simulation of depression and anxiety. Dialogues Clin. Neurosci. 8, 323-333.

Griebel, G., Simiand, J., Serradeil-Le Gal, C., Wagnon, J., Pascal. M., et al. (2002a). Anxiolytic- and antidepressant-like effects of the non-peptide vasopressin Vlb receptor antagonist, SSR149415, suggest an innovative approach for the treatment of stressrelated disorders. Proc. Natl. Acad. Sci. U.S.A. 99, 6370-6375.

Griebel, G., Simiand, J., Steinberg, R., Jung, M., Gully, D., et al. (2002b). 4- (2-Chloro-4-methoxy-5 methylphenyl)- $N$-[(1S)-2-cyclopropyl-1-(3-fluoro-4-methylphenyl) ethyl ]5-methyl- $N$-(2-propynyl)1,3 thiazol-2-amine hydochloride (SSR125543A), a potent and selective corticotrophin-releasing factor(1) receptor antagonist II. Characterization in rodent models of stress-related disorders. Pharmacol. Exp. Ther. 30, 333-345.

Henn, F.A., and Vollmayr, B. (2005). Stress models of depression: forming genetically vulnerable strains. Neurosci. Biobehav. Rev. 29, 799-804.

Hettema, J. M. (2008). The nosologic relationship between generalized anxiety disorder and major depression. Depress. Anxiety 25, 300-316.

Kalueff,A.V., Wheaton, M., and Murphy, D. L. (2007). What's wrong with my mouse model? Advances and strategies in animal modeling of anxiety and depression. Behav. Brain Res. 179, 1-18.

Kompagne, H., Bárdos, G., Szénási, G., Gacsályi, I., Hársing, L. G., et al. (2008). Chronic mild stress generates clear depressive but ambiguous anxiety-like behaviour in rats. Behav. Brain Res. 192, 311-314.

Kopp, C., Vogel, E., Rettori, M. C., Delagrange, P., and Misslin, R. (1999). The effects of melatonin on the behavioral disturbances induced by chronic mild stress in $\mathrm{C} 3 \mathrm{H} / \mathrm{He}$ mice. Behav. Pharmacol. 10, 73-83.

Li, S., Wang, C., Wang, W., Dong, H., Hou, P., et al. (2008). Chronic mild stress impairs cognition in mice: from brain homeostasis to behavior. Life Sci. 82, 934-942.

Maslova, L. N., Bulygina, V. V., and Markel, A.L. (2002).Chronicstress during prepubertal development:immediate and long-lasting effects on arterial blood pressure and anxiety-related behavior. Psychoneuroendocrinology 27, 549-561.

Mathews, K., Christmas, D., Swan, J., and Sorrel, E. (2005). Animal models of depression: navigating through the clinical fog. Neurosci. Biobehav. Rev. 29, 503-513.

Mergl, R., Seidscheck, I., Allgaier, A. K., Möller, H. J., Hegerl, U., et al. (2007). Depressive, anxiety, and somatoform disorders in primary care: prevalence and recognition. Depress. Anxiety 24, 185-195.

Olivier, J. D., Van Der Hart, M. G., Van Swelm, R. P., Dederen, P. J., Homberg, J. R., et al. (2008). A study in male and female 5-HT transporter knockout rats: an animal model for anxiety and depression disorders. Neuroscience 152, 573-584.

Orsetti, M., Colella, L., Dellarole, A. Canonico, P. L., and Ghi, P. (2007). Modification of spatial recognition memory and object discrimination after chronic administration of haloperidol, amitriptyline, sodium valproate or olanzapine in normal and anhedonic rats. Int. J. Neuropsychopharmacol. 10, 345-357.

Porsolt, R. D. (2000). Animal models of depression: utility for transgenic research. Rev. Neurosci. 11, 53-58.

Silva, R., Mesquita, A. R., Bessa, J., Sousa, J. C., Sotiropoulos, I., et al. (2008).Lithium blocks stress-induced changes in depressive-like behavior and hippocampal cell fate: the role of glycogen-synthase-kinase-3beta. Neuroscience 152, 656-669.

Song, C., and Leonard, B. E. (2005). The olfactory bulbectomised rat as a model of depression. Neurosci. Biobehav. Rev. $29,627-647$.

Song, L., Che, W., Min-Wei, W., Murakami, Y., and Matsumoto, K. (2006). Impairment of the spatial learning and memory induced by learned helplessness and chronic mild stress. Pharmacol. Biochem. Behav. 83, 186-193.

Sotiropoulos, I., Cerqueira,J.J.,Catania, C. Takashima, A., Sousa, N., et al. (2008). Stress and glucocorticoid footprints in the brain - the path from depression to Alzheimer's disease. Neurosci. Biobehav. Rev. 32, 1161-1173.

Sousa, N., Almeida, O. F., and Wotjak, C. T (2006). A hitchhiker's guide to behavioral analysis in laboratory rodents. Genes Brain Behav. 5, 5-24.

Steckler, T. (2001). The molecular neurobiology of stress - evidence from genetic and epigenetic models. Behav. Pharmacol. 12, 381-427.

Strekalova, T., Spanagel, R., Bartsch, D. Henn, F. A., and Gass, P. (2004). Stress-induced anhedonia in mice is associated with deficits in forced swimming and exploration.
Neuropsychopharmacology 29, 2007-2017.

Swaab, D. F., Bao, A. M., and Lucassen, P. J. (2005). The stress system in the human brain in depression and neurodegeneration. Ageing Res Rev. 4, 141-194.

Võikar, V., Kõks, S., Vasar, E., and Rauvala, H. (2001). Strain and gender differences in the behavior of mouse lines commonly used in transgenic studies. Physiol. Behav. 72, 271-281.

Willner, P. (2005). Chronic mild stress (CMS) revisited: consistency and behavioral-neurobiological concordance in the effects of CMS. Neuropsychobiology 52, 90-110.

Willner, P., and Mitchell, P. J. (2002). The validity of animal models of predisposition to depression. Behav. Pharmacol. 13, 169-188.

Willner, P., Moreau, J. L., Nielsen, C. K., Papp, M., and Sluzewska, A. (1996) Decreased hedonic responsiveness following chronic mild stress is not secondary to loss of body weight. Physiol. Behav. 60, 129-134.

Conflict of Interest Statement: The authors declare that the research was conducted in the absence of any commercial or financial relationships that could be construed as a potential conflict of interest.

Received: 12 September 2008; paper pending published: 21 October 2008; accepted: 12 January 2009; published online: 27 January 2009.

Citation: Bessa JM, Mesquita AR, Oliveira M, Pêgo JM, Cerqueira JJ, Palha JA, Almeida OFX and Sousa N (2009) A trans-dimensional approach to the behavioral aspects of depression. Front. Behav. Neurosci. (2009) 3:1. doi: 10.3389/neuro.08.001.2009

Copyright $\odot 2009$ Bessa, Mesquita, Oliveira, Pêgo, Cerqueira, Palha, Almeida and Sousa. This is an open-access article subject to an exclusive license agreement between the authors and the Frontiers Research Foundation, which permits unrestricted use, distribution, and reproduction in any medium, provided the original authors and source are credited. 